\title{
Advanced microscopic approaches to the study of eukaryotic chromosomes
}

\author{
Federica Borsatti and Mauro Mandrioli* \\ Dipartimento di Biologia Animale, Università di Modena e Reggio Emilia, Via Campi 213/D, 41100 Modena, Italy. \\ E-mail: mandrioli.mauro@unimore.it.
}

\begin{abstract}
Since the first discoveries of Schneider and Flemming, several papers have been published on chromosome structure making the understanding of chromatin organization in chromosomes one of the most attractive topics of the current biosciences. Several new discoveries on chromosome structure arose from advanced microscopy techniques that flanked light and electron microscopy. In the present review, the newest microscopy techniques applied to the study of the chromosome structure are presented and discussed in order to evidence the advantages and disadvantages of each approach.
\end{abstract}

Key words: atomic force microscopy, chromosome structure, confocal microscopy, electron tomography.

\section{INTRODUCTION}

The understanding of chromatin organization in chromosomes is one of the most attractive topics of the current biosciences.

DNA is packed into chromosomes by specialized proteins so that the $2 \mathrm{~nm}$-thick DNA fiber is compacted about 40 times into a $30 \mathrm{~nm}$ thick chromatin fiber. The attendant proteins in this process are the histones. DNA winds a core of histones formed by two copies of $\mathrm{H} 2 \mathrm{~A}, \mathrm{H} 2 \mathrm{~B}, \mathrm{H} 3$ and $\mathrm{H} 4$ histones respectively at intervals of around 200 nucleotide in which 146 base pairs (bp) wrap the histone core and $50 \mathrm{bp}$ act as a linker between the histone cores. These units, called nucleosomes, adopt a beads-on-string form and they are finally compacted together by $\mathrm{H} 1$ histones, which pull together the nucleosomes into a regular repeating array (for a review see SuMnER 2004). The discovery of nucleosomes as oligomers of histones on the DNA fiber provided a molecular description of the fundamental unit of chromatin folding and accounted for the first 6- to 7-fold linear compaction of DNA (EHRENHOFER-MURRAY 2004).

The next level of folding is the so-called " 30 $\mathrm{nm}$ " fiber. Although there has been significant controversy surrounding the description of this structure, the $30 \mathrm{~nm}$ fiber is usually seen as either

\footnotetext{
* Corresponding author: phone (+39) 59 2055544; fax (+39) 59 2055548; e-mail: mandriol@unimo.it
}

a coiling or folding of the nucleosomal fiber that generates another 6- to 7-fold compaction. In the mitotic state, the $30 \mathrm{~nm}$ fiber must compact another 200- to 500 -fold to achieve the final 10,000to 20,000-fold linear compaction of the mitotic chromosome (for a review see SUMNER 2004). These so-called higher-order levels of chromatin folding have proven as delicate as they are controversial. A classical view suggests a folding or helical coiling of the $30 \mathrm{~nm}$ fiber into increasingly larger structures (SwEDLOW and Hirano 2003 and reference within).

The successive levels of chromatin compaction have not been clearly elucidated even if there are several evidences that a hierarchy of chromatin loops and coils is responsible for the final chromosome structure (SwEDLOW and HiRANo 2003). In view of the complexity of higher order structures of chromosomes, many Authors tried to make the path of DNA visible within the chromosome by selectively solubilizing chromosomal proteins and/or by swelling the chromosome. For instance, the classic high-salt extraction of chromosomes produced a structure containing a proteinaceous axial core decorated by a series of DNA loops (Swedlow and HiRANo 2003). Alternatively, chromosomes can be simply swollen in a hypotonic buffer to visualize the location of chromosome components (e.g., EARNSHAW and LAEMMLi 1983, 1984; EARNSHAW and HeCK 1985). These early studies suggested the presence of a chromosome scaffold that determines the rod 
shape of a chromosome. The scaffold may be either an axial core, presumably made of DNA and/ or protein, that serves as the backbone of the chromosome (WANNER and FORMANEK 2000) or a loose network of DNA/protein complexes whose regulated assembly controls the higher-order structure of chromosomes (EARNSHAw and HECK 1985).

At the end of the compaction process, the DNA fiber is folded in order to achieve an end-toend compaction of $10,000-20,000$ fold that is a critical step in chromosome segregation, which is required for accurate transmission of genetic information during cell division. In a eukaryotic cell, the compaction of DNA into chromosomes is essential for the proper formation of the kinetochore and for ensuring that chromosome arms are short enough to be completely separated before cytokinesis (PORTER et al. 2004).

In order to go in depth into the analysis of chromosome structure several experimental approaches have been applied but the traditional tool used by cytogenetists for chromosome studies has been the microscope and in particular light microscope (LM) that allowed the analysis of chromosomes with a minimum of preparation. However, LM is limited in the information that could be obtained in view of the Abbe's law constrain on resolution.

Significant advances in the comprehension of chromosome architecture have been made using different microscopy techniques characterized by an increased resolving power. In particular, several papers reported the use of scanning electron microscopy (SEM) and transmission electron microscopy (TEM) to study chromosome structure at an ultra-structural level (XU and Wu 1983; Adolph and Kreisman 1985; Troster et al. 1985; Adolph et al. 1986; JaCK et al. 1986; Allen et al. 1986; Sumner and Ross 1989; Sumner 1991; Sumner et al. 1994; SQuARzoni et al. 1994; SumNER 1996; Wolf and SuMNER 1996; SuMNER 1998; Jenkins et al. 2002). However, these techniques required several experimental steps that could make difficult the analysis of chromosome structure and present some risks of artefacts (Sumner and Ross 1989; SAnChez-Sweatman et al. 1993).

In order to overcome these difficulties, several new microscope techniques have been developed in the last decades. In particular, the advent of different high-resolution microscopes allowed a better understanding of the higher levels of chromatin compaction making possible an improved comprehension of chromosome structure. This result is particular intriguing since it is becoming increasingly clear that chromatin higher-order structure (i.e. organization beyond the level of the linear array of nucleosomes) plays a critical role in many aspects of gene regulation (WoODCOCK and Dimitrov 2001).

However, a full understanding of these manifestations of chromatin higher-order structure and their functional significance requires the knowledge of the 3D arrangement of chromosomal components and the mechanisms and dynamics of their assembly and disassembly. At this regards, some new microscopy techniques resulted highly interesting and promising for the study of chromosome structure. In the present review, these newest microscopy techniques applied to the study of chromosome architecture are described and discussed.

Atomic force microscopy of eukaryotic chromosomes - In order to better understand chromosome structure at a nanometer scale several laboratories used SEM and TEM microscopes. However, in order to make ultra-structural analysis without difficult experimental steps, a new family of microscopes, called scanning probe microscopy (SPM), has been developed over the last 10 years (WIESENDANGER 1994). These microscopes operate by scanning a probe in an $\mathrm{x}$-y raster over the surface of the specimen. On the basis of the precise nature of the probe, different characteristics of the surface could be recorded. The local probe may be a conducting tip, as in scanning tunnelling microscopy (STM) (BINNIG et al. 1982; BoNNELl 1993), or a tapered etched optical fibre, as in scanning near field optical microscopy (SNOM) (BETZIG and TRAUTMANN 1992).

To date the SPM microscope most widely used in the study of biological specimens has been the atomic force microscopy (AFM), invented by BINNIG et al. (1986), since it provided new opportunities for imaging different biological samples, including chromosomes (YANG et al. 1993). In AFM, a sample is scanned in an $\mathrm{x}-\mathrm{y}$ raster below an ultra-sharp tip (with a radius of curvature of about $10 \mathrm{~nm}$ ), which is attached to a soft forcesensitive cantilever (with a typical length and spring constant of about $100 \mu \mathrm{m}$ and $1 \mathrm{~N} / \mathrm{m}$ respectively). Feedback control of the cantilever deflection at each $x-y$ position means that a direct, ultra-thin resolution 3D map of the sample is obtained.

The AFM uses the attractive or repulsive forces encountered by a probe tip when it is in close proximity to a sample surface $(<200 \mathrm{~nm})$. In 
particular, there are three main modes of AFM operation that are currently in use: Contact, NonContact and Intermittent Contact (Tapping). Contact AFM is done by bringing the tip to a distance at which repulsive forces dominate tip-sample interaction. The contact mode (where the tip scans the sample in close contact with the surface) is the common mode used in the force microscope. The force on the tip is repulsive with a mean value of $10^{-9} \mathrm{~N}$. This force is set by pushing the cantilever against the sample surface with a piezoelectric positioning element. Problems with contact mode are caused by excessive tracking forces applied by the probe to the sample that can result in sample damaging. These effects can be reduced by minimizing tracking force of the probe on the samples, but there are practical limits to the magnitude of the force that can be controlled by the user during operation in ambient environments. Under ambient conditions, sample surfaces are covered by a layer of adsorbed gases consisting primarily of water vapour and nitrogen, which is 10-30 mono-layer thick. When the probe touches this contaminant layer, a meniscus forms and the cantilever is pulled by surface tension toward the sample surface. The magnitude of the force depends on the details of the probe geometry, but is typically on the order of 100 nanoNewtons. This meniscus force and other attractive forces may be neutralized by operating with the probe and part or all of the sample totally immersed in liquid. There are many advantages to operate AFM with the sample and cantilever immersed in a fluid that include the elimination of capillary forces, the reduction of Van der Waals' forces and the ability to study technologically or biologically important processes at liquid solid interfaces. However, there are also some disadvantages involved in working in liquids. These range from nuisances such as leaks to more fundamental problems such as sample damage on hydrated and vulnerable biological samples.

An attempt to avoid these problems is the Non-contact Mode that is done bringing the tipsample interaction is in the attractive or van der Waals regime. In this mode the tip hovers 50 - 150 Angstrom above the sample surface. Attractive Van der Waals forces acting between the tip and the sample are detected and topographic images are constructed by scanning the tip above the surface. Unfortunately the attractive forces from the sample are substantially weaker than the forces used by contact mode. For highest resolution, it is necessary to measure force gradients from Van der Waals forces, which may extend only a na- nometer from the sample surface. In general, the fluid contaminant layer is substantially thicker than the range of the Van der Waals force gradient and therefore, attempts to image the true surface with non-contact AFM fail as the oscillating probe becomes trapped in the fluid layer or hovers beyond the effective range of the forces it attempts to measure. In the Non-Contact AFM operating mode the force is measured by comparing the frequency and/or amplitude of the cantilever oscillation relative to the driving signal.

Tapping or Intermittant Contact mode represents a key advance in AFM. This potent technique allows high-resolution topographic imaging of sample surfaces that are easily damaged, loosely hold to their substrate or difficult to image by other AFM techniques. Tapping mode overcomes problems associated with friction, adhesion, electrostatic forces and other difficulties that plague conventional AFM scanning methods by alternately placing the tip in contact with the surface to provide high resolution and then lifting the tip off the surface to avoid dragging the tip across the surface. Tapping mode imaging is implemented in ambient air by oscillating the cantilever assembly at or near the cantilever's resonant frequency using a piezoelectric crystal. The piezo motion causes the cantilever to oscillate with a high amplitude (typically greater than $20 \mathrm{~nm}$ ) when the tip is not in contact with the surface. The oscillating tip is then moved toward the surface until it begins to lightly touch, or tap, the surface. During scanning, the vertically oscillating tip alternately contacts the surface and lifts off, generally at a frequency of 50,000 to 500,000 cycles per second. As the oscillating cantilever begins to intermittently contact the surface, the cantilever oscillation is necessarily reduced due to energy loss caused by the tip contacting the surface. The reduction in oscillation amplitude is used to identify and measure surface features.

The cantilever oscillation amplitude is maintained constant during tapping mode operation by a feedback loop. Selection of the optimal oscillation frequency is software-assisted and the force on the sample is automatically set and maintained at the lowest possible level. When the tip passes over a bump in the surface, the cantilever has less room to oscillate and the amplitude of oscillation decreases. Conversely, when the tip passes over a depression, the cantilever has more room to oscillate and the amplitude increases (approaching the maximum free air amplitude).

Tapping mode operation in fluid has the same advantages as in the air or vacuum. However im- 
aging in a fluid medium tends to damp the cantilever's normal resonant frequency. In this case, the entire fluid cell can be oscillated to drive the cantilever into oscillation. This is different from the tapping or non-contact operation in air or vacuum where the cantilever itself is oscillating. When an appropriate frequency is selected (usually in the range of 5,000 to 40,000 cycles per second), the amplitude of the cantilever will decrease when the tip begins to tap the sample, similar to Tapping Mode operation in air. Alternatively, the very soft cantilevers can be used to get the good results in fluid. The spring constant is typically 0.1 $\mathrm{N} / \mathrm{m}$ compared to the tapping mode in air where the cantilever may be in the range of $1-100 \mathrm{~N} / \mathrm{m}$.

In view of these properties, Intermittent contact mode results extremely interesting in order to reduce the risk of damaging potentially fragile samples, such as biological ones (Fotiadis et al. 2002; Giocondi et al. 2003).

The concept of resolution in AFM is different from radiation-based microscopes because AFM imaging is a $3 \mathrm{D}$ dimensional imaging technique. The ability to distinguish two separate points on an image is the standard by which lateral resolution is usually defined. There is clearly an important distinction between images resolved by wave optics and scanning probe techniques. The former is limited by diffraction and later primarily by apical probe geometry and sample geometry. Usually the width of a DNA molecule is loosely used as a measure of resolution, because it has a known diameter of $2.0 \mathrm{~nm}$ in the B form. Some of the best values for AFM imaging are $3.0 \mathrm{~nm}$ quoted form DNA in propanol. Unfortunately, this definition of resolution can be misleading because the sample height clearly affects this value (Fotiadis et al. 2002; Giocondi et al. 2003).

The magnification power achieved by atomic force microscopes rivals that of transmission and scanning electron microscopes. Atomic force microscopes also are capable of a wide field of view (similar to SEM) and extreme vertical resolution (like TEM). The ratio of vertical to horizontal magnification can exceed 1,000:1, permitting the discrimination of subtle differences between extremely smooth surfaces; when combined with phase imaging, this can facilitate the discernment of differences in the chemical composition of sample surfaces. In general, AFM illuminates topographic contrasts, permits atomic scale measurements and provides for the analysis of unmodified surface features (i.e., the sample need not be coated as in electron microscope technology) without the extensive effort, time and resources needed to prepare samples for other technologies. Thus, atomic force microscopy is a very powerful and cost-efficient tool to employ in biological research (Fotiadis et al. 2002; Giocondi et al. 2003).

Interesting peculiarities of AFM are that specimens can be examined without any preparatory treatment and that the examining of biological materials can be performed in air or under physiological conditions rather than under vacuum $(\mathrm{DE}$ Grooth and Putman 1992; Rasch et al. 1993; McMaster et al. 1994). AFM has been used to image a wide range of biological samples that include single molecules, such as DNA, cells and tissues (EngEl 1991; Bustamante et al. 1994).

An important feature of AFM in cytogenetics is that standard chromosome preparations can be examined without any additional treatment. The potential of AFM for high-resolution analysis of chromatin has been repeatedly reported from the beginning of ' 90 by various Authors that worked with both plant and animal chromosomes, including human ones (De Grooth and Putman 1992; RASCH et al. 1993; McMaster et al. 1994; WINFIELD et al. 1995).

Chromosomal samples for AFM analysis are generally obtained by squashes or spreading techniques (WINFIELD et al. 1995). A prerequisite is the obtaining of chromosome spreads with a minimum of overlaying cellular debris and cytoplasmatic material, which would have obscured ultrastructural detail. At this aim, several Authors (especially in the case of plant materials) treated chromosomes with hot $45 \%$ acetic acid to clean chromosome samples from cytosol material that could cover chromosomes and interfere with the observation of chromosomal surface (SCHAPER et al. 2000; Sugryama et al. 2003). Sugiyama and colleagues evidenced in 2004 that the three-dimensional (3D) structure of chromosome was damaged and the alignment of chromatin fibers on chromosome surface disordered after acetic acid treatment. This structural disorder was due to the significant protein extraction from the chromosomes caused by acetic acid (SUGIYAMA et al. 2004).

Phase contrast microscope is generally used to aid the selection of suitable spreads for further AFM imaging. AFM analysis of chromosomes is realized operating in contact mode of imaging working in air at room temperature at a constant force between tip and sample of about 1-10 $\mathrm{nN}$.

AFM permits to clearly resolve chromosomes or portions of chromosome including nucleolar 
organizing regions and centromeres (MCMASTER et al. 1996a).

After 3D reconstruction, centromeres of monocentric chromosomes resulted as a depression between the two chromosomal arms (McMASTER et al. 1996a). Interestingly, just one paper on AFM analysis of holocentric chromosomes has been published (MANDrioli and MANICARDI 2003). 3D reconstruction of AFM imaged insect holocentric chromosomes clearly evidenced that the junction between the two chromatids is absolutely homogenous along the entire chromosome length and it appeared as a deep depression between the two chromatids. AFM analysis indicated that holocentric chromatids adhere to one another without any interruption or prominent additional material between them. AFM analysis, therefore, provided new and reliable evidences at a nanomolecular level concerning the holocentric structure of insect holokinetic chromosomes (Mandrioli and Manicardi 2003).

AFM observation at high magnification of plant chromosomes revealed several chromosomal surface features of different length scales, the smallest of which are in the size range $10-20 \mathrm{~nm}$. The origin of these features has not been assessed although their sizes are in agreement with nucleosome structures (WINFIELD et al. 1995; McMasTER et al. 1996a). SEM analysis of plant chromosomes also furnished indications of nucleosomes (WANNER et al. 1991; Sugryama et al. 2003).

Analysis of untreated human chromosomes with AFM revealed a pattern of alternating thicker and thinner bands (TAmayo and Miles 2000; Musio et al. 1994; Ushiki et al. 2002; TAmayo 2003a, b). A comparison of the height profiles obtained at AFM with the results of the conventional banding techniques showed that $G$ and $\mathrm{R}$ bands corresponded to the alternating thicker and thinner bands respectively (TAMAYO 2003b). In particular, the very low gene density at $G$ bands could explain the higher packing of these regions on the contrary of what happens in the thinner R bands where DNA should be accessible to the transcription and replication machinery.

The best agreement of AFM banding resulted with the highest resolution ideogram (850 bands) obtained digitizing $G$ banded and stained prometaphase chromosomes with a low degree of condensation (FRANCKE 1994; HARNDEN and KLINGER 1985). Interestingly, this result has been showed on metaphase chromosomes, whose degree of condensation was determined between 400 and 550 bands by standard banding techniques (TAmayo 2003b). These data, as a whole, indicated that AFM is a powerful tool for diagnosis technique since it allowed the visualization of bands at high resolution in chromosomes without any staining or banding.

$\mathrm{N}$ and $\mathrm{C}$ banded chromosomes have also been observed and in all cases positive bands appeared as areas of high relief with heights of up to 150$300 \mathrm{~nm}$ greater than negative unbanded regions. These results suggested a sort of collapse of chromosome structure so that, after banding, chromosomes have a distinct ridge around their periphery and a collapse centre.

Several Authors indicated that it is possible to identify fluorescent in situ hybridisation (FISH) signals with AFM even if not all signals observed with the LM appeared as areas of high relief after AFM imaging since only the larger signals were imaged effectively (RAsch et al. 1993; MCMASTER et al. 1996a). These results could be explained considering the physical properties of the reporter molecule employed for detection (generally fluorescein isothiocyanate) in FISH experiments. At this regard, RASCH et al. (1993) reported that hybridisations performed using 3,3-diaminobenzidine $(D A B)$ as a reporter were able to evidence in AFM several bands that have not been visualized at LM including small signals separated by only $500 \mathrm{~nm}$.

AFM has been also applied for karyotype construction that has been obtained using chromosome volumes as a parameter for homologue identification (McMaster et al. 1996b).

Finally, AFM can be used to manipulate biological materials with relative ease and at high resolution. In particular, some Authors indicated that AFM can be used for nanomanipulation and nanoextraction of DNAs that could be used to generate probe sets specific for subregions of the genome and useful for the painting of chromosome bands. In particular, probes could be produced by PCR amplification of AFM-dissected chromosome regions (ThalHAMmer et al. 1997; IwaBuchil et al. 2002; StARK et al. 2003). Compared to standard microdissection techniques, the AFM can be used with much higher precision for the dissection of the region of interest and subsequent nanoextraction of DNA material. After scanning the area of interest in non-contact mode AFM, chromosome bands could be cut by the AFM tip at high force. The genetic material of a single cut attached to the tip can be extracted and amplified using degenerate oligonucleotideprimed-PCR. Subsequent to hapten labelling, fluorescence in situ hybridization can be performed and chromosome band-specific probes 
visualized by standard fluorescence microscopy (Thalhammer et al. 1997; Iwabuchil et al. 2002; STARK et al. 2003). Cross-sectional analysis of images obtained with AFM-derived probes revealed that AFM cuts between $70 \mathrm{~nm}$ and $280 \mathrm{~nm}$ whereas a 380-nm-wide UV-laser cut was obtained using a microscope equipped for ultraviolet (UV) microbeam laser manipulation (STARK et al. 2003).

In view of these properties, AFM is a powerful tool for cytogenetists since it can be applied not only to analyse chromosome structure but also to generate specific genomic probes.

Three-dimensional reconstruction of chromosomes through electron tomography - Three-dimensional (3D) structure of chromosomes is far beyond the reach of ordinary light or confocal microscopes. On the contrary, the electron tomography method (ETM), i.e. transmission electron microscopy (TEM) tomography, is able to provide information regarding both qualitative and quantitative spatial organization of macromolecules. In particular, important improvements derived by the replacing of TEM with ETM.

TEM images are produced using orthogonal projections of the three dimensional object under study. Tilt series of image projections of an object from $0^{\circ}$ to $\pm 60^{\circ}$ are recovered with a eucentric goniometer specimen holder that is usually incorporated in the modern TEM instruments. The theoretical resolution of TEM at $100 \mathrm{kV}$ is 0.003 $\mathrm{nm}$, which is sufficient for visualizing atoms (for example, the van der Waals radiuses of hydrogen and carbon atoms are 0.12 and $0.2 \mathrm{~nm}$ respectively). In practice, in view of some instrumental constraints, the resolution of modern TEM is at the best $0.1 \mathrm{~nm}$, which still is at an atomic level. However, this resolution power is not reliable for biological samples that could be analysed with a theoretical resolving power that is not better than 1-2 $\mathrm{nm}$. At least partly, this reduction in resolution is due to the fact that details in images are in variable degrees superimposed owing to specimen thickness.

Electron tomography is a general method for three-dimensional reconstruction of single, transparent objects from a series of projection images (i.e. from a tilt series) recorded with a transmission electron microscope. Electron tomography is not restricted to symmetrical objects or regularly arranged objects, nor to objects with preferred orientations on a support grid. So far, the electron tomography method covers the intermediate resolution range of 2.5-5.0 nm. Data obtained with electron tomography furnish a rich source of quantitative information about the structural composition and organization of cellular components. It offers the opportunity to obtain $3 \mathrm{D}$ information on structural cellular arrangements with a significant higher resolution than that provided by any other method currently available (AUER 2000).

The set of projections used in ETM are named Radon transforms in view of the name of the $\mathrm{Au}$ thor that firstly presented the mathematical method of rebuilding the original 3D object from its projections (RADON 1917; for a translation of the paper see Deans 1983). Despite this method was developed at the beginning of the ninetieth century (1917), the first 3D reconstructions of biological samples have been published only in 1968 (De Rosier and Klug 1968; Hart 1968; Hoppe et al. 1968). Chromosome studies played a pivotal role in the development of ETM as assessed by the fact that the first successful tomographic reconstruction is of a whole-mounted chromosome (Harautz et al. 1987; Frank 1992).

Major problems with this technique arise from electron dose limitation considerations, a limited tilting range, angular under-sampling and alignment errors. More formally, the problem is to reconstruct a 3D signal out of noisy, incomplete and using indirect observations in 2D. Finally, ETM has not yet become a widely used tool in structural biology since it represents a time-consuming method. However, the availability of high-performance computing and instruments makes today easier to apply ETM analysis so that this technique is interesting in order to have a closer look to higher order structure of chromosomes that have so far been almost impenetrable (ENGELHARDT 2000).

Despite these interesting assumptions, some troubles are still present as indicated by the fact that at the moment there is no consensus about the preparation steps for chromosome samples for ETM investigations (WoodCock 1992). To date, most ordinary preparation methods used in TEM investigations (such as sections of epoxy resin embeddings, whole mounts and cryo-EM samples) are usually also convenient in ETM (WoodCock 1992; Horowitz et al. 1997). Nevertheless, ETM revealed shortcomings in the use of some common preparative methods that were responsible of flattened or unsuccessful 3D reconstructions (ENGELHARDT 2000). An example is represented by sections of epoxy resin embeddings since reconstructions obtained by section analysis may suffer in view of deformations during cutting 
(ENGELHARDT 2000). Whole mounts are preferable for achieving a high signal-to-noise ratio and to secure that the whole content of the object under investigation is carried along. This is not always the situation for ultrathin sections. Sections of large organelles such as eukaryotic chromosomes and nuclei can, however, be of advantage when specimens such as whole mounts are too thick for ordinary 100-120-kV TEM; in earlier studies it was considered that $1 \mathrm{MV}$ was the only choice for chromosomes. Today, with modifications of preparative methods, $100-120-\mathrm{kV}$ EM has proved sufficient for whole mounts, not only for whole-mounted chromosomes, but also various parts of whole-mounted cells.

In order to apply ETM for cytogenetic studies, chromosome are usually post-fixed with glutaraldehyde (GA) with the aim of preserving chromosome coiling for $3 \mathrm{D}$ reconstruction (ENGELHARDT 2000).

After fixation, chromosomal preparations have to be dried. Air-drying causes artefacts, shrinkage, wrinkling and distortion that alter chromosome coiling and structure. Drying artefacts are due to the disruptive effects of the surface tension of the fluids from which samples are dried. This problem has been solved with the critical point drying (CPD) method (that is conventionally used in SEM) even if some disadvantages, such as thermal and pressure stresses onto samples, may be difficult to avoid (Sugryama et al. 2004). Alternative techniques are the tert-butanol method and the freeze-drying (Sugiyama et al. 2004).

After sample preparation, ETM analysis is performed recording tilt series of images (manually or using an automatic recording function). The tilt-series images collected must be most accurately aligned to be sufficient for ETM reconstructions, especially after manual recording and scanning procedures. Alignment of the tilt series can be accomplished without markers by using the cross-correlation function method as used in the automatic data collection process. However, before the cross-correlation or other automatic method has been better refined, the use of fiducial markers has proved to be a more accurate method for the aligning of tilt series data (FRANK 1992; ENGELHARDT 2000).

Images obtained after alignment can be contrast-enhanced, temporarily or permanently, enlarged and moved to different regions when the image size exceeds the display and processed otherwise, e.g. with unsharp masking and other filters.
The ETM reconstruction programs that are used need a set of accurately aligned images, i.e. Radon transforms. Some of the used algorithms are the algebraic reconstruction technique (ART), the multiplicative algebraic reconstruction technique (MART), the maximum entropy method (MEM) and the weighted back-projection method (WBM). At the moment, no single algorithm can be considered as the best since some algorithms that construct well in one case do poorly in others. In order to solve this problem, some Authors suggested the use of at least two different algorithms; for instance reconstruction could be first produced, as a test, with the WBM, then, if the reconstruction with WBM shows adequate results, the reconstruction could be completed with the MEM (as suggested by ENGELHARDT 2000). The main disadvantage of MEM for ETM is that it is calculation intensive. However, this match is not very valid for long, as the computers are steadily upgraded and now the time needed for MEM processing is acceptable. MEM was first introduced in an astrophysical context for filtering telescope pictures from noise, and the method initially presented significant improvements in image quality. The introduction into $3-\mathrm{D}$ reconstruction procedures in structural biology was accomplished for the first time for chromosome studies, as earlier mentioned. After this initial work, relatively few investigators in ETM have shown an interest in using MEM. This must be partly due to a lack of computing power and long processing times, or to poor evaluation methods used in viewing $3-\mathrm{D}$ reconstructions. The disadvantage with MEM is the long calculation time needed for large volumes (e.g. 400_400_500 pixels), only manageable for laboratories having access to supercomputer facilities. However, even desktop computers are today as fast as yesterday's supercomputers, so it should not be long before any laboratory will have the capacity to perform such computing. For smaller volumes (e.g. 100-100100 pixels) with an ordinary desktop computer this might already, or soon, be possible within a reasonable performance time (ENGELHARDT 2000).

The ART and MART algorithms are conceptually simple and computationally efficient, which make them good general-purpose algorithms. The ART algorithm used to reconstruct the images seeks to minimize the root mean square difference between the observed total electron content (TEC) data and those computed from the reconstruction. The ART algorithm is iterative and requires some starting image as an initial guess. The 
algorithm computes the root mean squared difference at each iteration and then makes additive changes that seek to minimize the difference. In many ways, MART is similar to ART. It is also iterative and starts from an initial guess. It compares TEC computed from the initial guess with the measured TEC. At each iteration, changes to the image are based on the difference. Of course, the difference with ART is that the changes are multiplicative rather than additive.

ETM furnished very interesting results about the $3 \mathrm{D}$ structure of chromatin fibers working on sections of nuclei (Horowitz et al. 1994; 1997). Low temperature embedding and nucleic acidspecific staining allowed individual nucleosomes to be clearly seen. Chromatin fibers showed complex 3D trajectories with smoothly bending regions interspersed with abrupt changes in direction and U turns. Nucleosomes are located predominantly at the fiber periphery, whereas linker DNA tends to project toward the fiber interior. Within the fibers, the unifying structural motif is a two nucleosome-wide ribbon that is variably bent and twisted and in which there is little face-to-face contact between nucleosomes (Horowitz et al. 1994; 1997).

On the basis of these results, Horowitz and colleagues proposed that this asymmetric $3 \mathrm{D}$ zigzag of nucleosomes and linker DNA represents a basic principle of chromatin folding that is determined by the properties of the nucleosome-linker unit. This concept of chromatin fiber architecture is in contrast with the helical models in which specific nucleosome-nucleosome contacts play a major role in generating a symmetrical higher order structure (Horowitz et al. 1994; 1997).

An asymmetrical 3D zig-zag of nucleosomes would generally present a more open substrate to the transcriptional regulatory machinery than helical models with strong nucleosome-nucleosome contacts. The transcriptional control implications of a more open and irregular chromatin structure should be therefore analysed in future works performed with ETM. The ability of ETM to obtain high quality 3D information from minimally perturbed specimens could be, in fact, essential to verify if it is appropriate to continue to view chromatin in terms of hierarchy of specific folding levels instead of verifying alternative models for chromosome and chromatin structures.

Other interesting applications of ETM applications for the study of chromosome structure regard the $3 \mathrm{D}$ reconstruction of nucleolar organizing regions (NORs). In particular, these papers showed that argyrophilic NOR proteins are grouped as a fiber of $60-80 \mathrm{~nm}$ in diameter that constitutes either one part of a turn or two or three turns of a helix within small and large double-spotted NORs, respectively. Within crescentshaped NORs, virtual slices reveal that the fiber constitutes several longitudinally twisted loops, grouped as two helical 250- to 300-nm coils, each centred on a non-argyrophilic axis of condensed chromatin. These data, as a whole, allowed the suggestion of a model of the 3D organization of chromatin within NORs, in which loops are twisted and bent to constitute one basic chromatid coil (Heliot et al. 1997; KLein et al. 1998).

The ultimate goal in $3 \mathrm{D}$ reconstruction studies with ETM would be to recognize different proteins, nucleic acids and other macromolecular assemblies straight from their structural 3D configuration. At this regards, interesting developments are represented by the immuno-electron tomography (IET) (SugIYAMA et al. 2004) and by the in situ hybridization electron tomography since they both allowed the obtaining of highresolution 3D structures of specific chromosomal regions (SugiYama et al. 2004).

Confocal analysis of chromosomes structure and $d y$ namics - Advances in computer technology and in sensitivity of optical imaging devices allowed the development of a new generation of instruments for microscopic examination of a wide variety of biological specimens that include laser-scanning confocal microscope (CM) (Cox 1993; WRIGHT et al. 1993; Boyde 1994; LichtMan 1994; OckLEFORD 1995).

$\mathrm{CM}$ utilizes a gas laser, a conventional light microscope, confocal optics, extremely sensitive light sensors and a computer equipped with software to produce images of exceptional clarity and resolution (Cox 1993; Wright et al. 1993; Boyde 1994; LichtMan 1994; OCKLEFord 1995). In addition, since information on an examined specimen is collected as volume data in digital format, a wide variety of computer-driven image-processing programs may be applied to further enhance the usefulness of the images. These features, as a whole, make $\mathrm{CM}$ an important improvement in respect to the classical LM and indicate that $\mathrm{CM}$ could be an intriguing tool to open the door to 3D analysis of cells and chromosomes at high-resolution (ROWLAND and NiCKLESS 2000).

In LM, the light from a source below the specimen is projected through a small illumination stop aperture and collected by a condenser lens that focuses the light on a specific volume element within the specimen. This light passes through the 
specimen and is collected by the objective lens of the microscope and focused at the image stop aperture. The image formed is either directly observed with ones eyes or it is collected by a device such as a video camera and projected on a video monitor.

In a confocal microscope out-of-focus light from a specimen being observed reaches a focal point at a different level relative to the focal point of the in-focus light. A small imaging aperture at the focal point of the in-focus light from the specimen will block most of the out-of-focus light rays, thus reducing out-of-focus interference in the observed image. If the aperture is small enough, nearly all the out-of-focus light may be eliminated; however, this greatly reduces the amount of light producing the image. So in classical microscopy, the degree of confocality would be limited by the need to have sufficient light to see the image. The term confocal means that both the illumination and the imaging components of the microscope are focused on the same volume element of the specimen (WRIGHT et al. 1993; STEVENS 1994).

In a confocal-laser-scanning-microscope, the excitation light beam is scanned across the imaged area of the specimen in a series of directly adjacent parallel lines and the image is collected in digital form (i.e. as a series of points making up these lines). The signals from the PMT are sent to an amplifier and then processed using a computer. This allows a confocal microscope to produce a highly magnified image with exceptional contrast and often with striking details that would be blurred in an image from a classical light microscope (WRight et al. 1993; SteVENS 1994).

Since all the data are collected in digitized form, it may be easily processed with appropriate computer software to improve clarity. In addition, since a confocal microscope effectively sees only one plane of focus for any given image, it is possible to collect a series of optical sections by scanning a series of focal planes at different depths within the specimen. Successively, these sections may be recombined to produce $3 \mathrm{D}$ representations either as rendered volumes in perspective or as stereo images that may be viewed with appropriate optics to give 3D images (WRIGHT et al. 1993; Stevens 1994; Rowland and Nickless 2000).

In view of these properties, most of the current 3D fluorescence microscopy is done using the confocal microscope. However, although confocal microscopy has many advantages, it does also have limitations. A serious drawback for some applications is the amount of excitation light re- quired to produce confocal images. This may be a problem for fixed specimens that require many focal-plane images or for fixed specimens that are labelled with several different dyes. In these cases, the excitation-light dosage required to obtain satisfactory 3D images may bleach the dye. This sensitivity issue is especially critical when living specimens are examined. In this case, specimen viability as well as bleaching become serious concerns (McNally et al. 1999).

These limitations in sensitivity placed some constraints on what can be accomplished by confocal microscopy, particularly for long-term 3D imaging of living specimens. Time-lapse studies are often of interest to observe changes in the distribution of a molecule or movements of organelles within a cell. Often it is desirable, if not essential, to follow these changes in $3 \mathrm{D}$, for example to track objects that move from one focal plane to another. In many applications, it is also advisable to collect many, closely spaced focal planes because this provides improved resolution of the image in the third dimension (Z). Ideally, $\mathrm{Z}$ resolution should be close to the resolution obtained within the focal plane, and so for high Z resolution, 100 or more focal planes might well be necessary to span the full depth of a specimen. All of these requirements add up to a considerable light dosage. A 3D time-lapse sequence of 50 time points, with 50 focal planes per time point, would require 2500 images (MCNALLY et al. 1999).

Such imaging requirements are met by a complementary approach to 3D microscopy referred to as deconvolution (also known as wide-field deconvolution, digital-imaging microscopy, digital confocal, computational optical sectioning, or exhaustive photon reassignment). Deconvolution provides a computational solution to this problem by calculating and subsequently subtracting the component of a fluorescence image that comes from planes above and below the plane of interest. A point spread function (PSF) is determined for each objective by measuring a fluorescent bead, which mathematically describes how fluorescent light from a single point will be diffracted. During deconvolution the Fourier transform of the PSF is applied to the raw data images to reassign the out of focus information back to the point where it was emitted. The result is a deconvolved image in which details can be distinguished more clearly. Deconvolution microscope systems are now cheaper than most confocal microscopes and collect data faster than most confocals. However, to produce a 3D image, the deconvolution approach requires computational processing that can take 
anywhere from seconds to hours. In addition, interpretation of these images requires some knowledge of the processing methods such that a user can both recognize artefacts and identify real features (McNally et al. 1999). In view of these assumptions, it is not easy to define whether widefield microscopy plus deconvolution is better than confocal microscopy. A possible reply depends upon the resilience of the specimen under exposure to light. Fragile live specimens will survive, in fact, longer with the lower light exposure of widefield microscopy, whereas confocal produces better contrast with thick specimens.

$\mathrm{CM}$ resulted highly interesting for the study of interphase nuclei and in particular to verify the organization of chromosomes within nuclei. CM has been, in fact, coupled with fluorescent in situ hybridisation (FISH) in order to study the distribution of chromatin from each chromosome at interphase showing that nuclei are in a very well-defined organization (SHAw et al. 2003). The same microscopy approach resulted extremely useful also in the study of architectural alterations of interphase chromatin after changes in DNA methylation and histone acetylation (SANTOS et al. 2002).

$\mathrm{CM}$ allowed not only the analysis of interphase chromatin but also the $3 \mathrm{D}$ arrangements of centromeres and telomeres with the ability of comparing telomere length between sister chromatids or among telomeres of different chromosomes isolated from the same cell (BEKAERT et al. 2002; WeIERICH et al. 2003).

A further structure that could be analysed using $\mathrm{CM}$ is microtubule distribution during meiosis and mitosis in order to study not only chromosomes structure but also chromosome dynamic and kinetic during cell division (Boiso et al. 2002; WiLson et al. 2003; Mochida et al. 2004).

A particularly interesting application derived from $\mathrm{CM}$ is the fluorescence resonance energy transfer (FRET) (Sekar and Periasamy 2003). FRET is a distance-dependent physical process by which energy is transferred non-radioactively from an excited molecular fluorophore (the donor) to another fluorophore (the acceptor) by means of intermolecular long-range dipole-dipole coupling. FRET can be an accurate measurement of molecular proximity at angstrom distances (10$100 \AA$ ) and highly efficient if the donor and acceptor are positioned within the Förster radius (the distance at which half the excitation energy of the donor is transferred to the acceptor, typically $3-6 \mathrm{~nm}$ ).
The efficiency of FRET is dependent on the inverse sixth power of intermolecular separation (Förster 1965; Clegg 1996; Lakowicz 1999) making it a sensitive technique for investigating a variety of biological phenomena that produce changes in molecular proximity (DOs REMEDIOs et al. 1987). In particular, the technological advances in light microscopy imaging, combined with the availability of genetically encoded fluorescent proteins and antibodies, provided new tools useful to obtain spatial and temporal distribution of protein associations inside living cells and chromosomes (HEIM and TsIEN 1996; DAY 1998; ElANGOVAN et al. 2002; 2003).

FRET microscopy relies on the ability to capture fluorescent signals from the interactions of labelled molecules in single living or fixed cells. If FRET occurs, the donor channel signal will be quenched and the acceptor channel signal will be sensitized or increased (HERMAN 1998). FRET microscopic imaging allows not only the co-localization of the donor- and acceptor-labelled probes within $0.09 \mathrm{\mu m}^{2}$, but also the analysis of the molecular associations at close distances.

Several FRET microscopy techniques exist, each with its own advantages and disadvantages. They are used for various biological applications, including studies of organelle structure, cytochemical identification and oxidative metabolism (Sekar and Periasamy 2003). Confocal microscopy, however, is limited to standard laser lines of defined wavelengths. MP-FRET microscopy overcomes this limitation by using a tunable laser (range 700-1000 nm), allowing excitation of a wide variety of fluorophores with higher axial resolution, greater sample penetration, reduced photobleaching of marker dyes and increased cell viability. These advantages allow investigations on thick living tissue specimens that would not otherwise be possible with conventional techniques. However, all of these intensity-based FRET techniques require processing software to remove the unwanted bleed-through components in the FRET image (SEkar and Perisamy 2003).

\section{CONCLUSIONS}

The idea of chromosomes only appeared in the last quarter of nineteenth century when Schneider and Flemming firstly described the process of mitosis and the structure of mitotic chromosomes in plants and animals respectively (SUMNER 2004). Their works formed the foundation of the modern 
cytogenetic studies on chromosomes structures and functions.

Since these first discoveries, several papers have been published but the highest levels of chromosome organization are still poorly understood. Nevertheless, good progresses have been made by cytogenetists through the use of the newest advanced microscopy techniques and much should be clearer in a few years' time.

In particular, the analysis of chromosomes with advanced microscopy technique indicated that chromosomes behave as a polymer brush suggesting the presence of helical coiling and radial looping as the last level of chromosome organization. These results, as a whole, are very intriguing but suggest that there is still much to be learnt about chromosome structure. In view of these assumptions, the structure of eukaryotic chromosomes will be one of the most important topics in biosciences also in this new century.

Acknowledgements- - We apologize to those colleagues whose works could not be cited because space limitation. This work was supported by grants from the University of Modena and Reggio Emilia (M.M.)

\section{REFERENCES}

Adolph K.W. and Kreisman L.R., 1985 - Relationship of the surface structure of metaphase chromosomes to the higher order organization of chromatin fibers. Scan. Electron. Microsc. Pt. 2, pp. 869-877.

Adolph K.W., Kreisman L.R. and KueHn R.L., 1986 - Assembly of chromatin fibers into metaphase chromosomes analysed by transmission electron microscopy and scanning electron microscopy. Biophys. J., 49: 221-231.

Allen T.D., Jack E.M., Harrison C.J. and Claugher D., 1986 - Scanning electron microscopy of human metaphase chromosomes. Scan. Electron. Microsc. Pt. 1, pp. 301-308.

Auer M., 2000 - Three-dimensional electron cryo-microscopy as a powerful structural tool in molecular medicine. J. Mol. Med., 78: 191-202.

Bekaert S., Koll S., Thas O. and Van Oostveldt P., 2002 - Comparing telomere length of sister chromatids in buman lymphocytes using three-dimensional confocal microscopy. Cytometry, 48: 34-44.

Betzig E. and Trautmann J.K., 1992 - Near field optics: microscopy, spectroscopy and surface modification beyond the diffraction limit. Science, 257: 189195.

Binning G., Rohrer H., Gerber C. and Weibel E., 1982 - Tunneling through a controllable vacuum gap. Appl. Phys. Lett., 40: 178-180.
Binning G., Quate C.F. and Gerber C., 1986 Atomic force microscope. Phys. Rev. Lett., 56: 930933.

Boiso I., Marti M., Santalo J., Ponsa M., Barri P.N. and Veiga A., 2002 - A confocal microscopy analysis of the spindle and chromosome configurations of buman oocytes cryopreserved at the germinal vesicle and metaphase II stage. Hum. Reprod., 17: 18851891.

BonNell D.A., 1993 - Scanning tunnelling microscopy and spectroscopy: theory, techniques and applications. VCH Publishers Inc (New York, USA).

Boyde A., 1994 - Bibliography on Confocal Microscopy and Its Applications. Scanning, 16:33-56.

Bustamante C., Erie D.A. and Keller D., 1994 Biochemical and structural applications of scanning force microscopy. Curr. Opin. Struct. Biol., 4: 750 760.

CleggG R.M., 1996 - Fluorescence resonance energy transfer. Fluorescence Imaging Spectroscopy and Microscopy. Vol. 137. X.F. Wang and B. Herman editors, John Wiley \& Sons Inc., New York. pp. 179251

Cox G., 1993 - Trends in Confocal Microscopy. Micron, 24: 237-247.

DAY R.N., 1998 - Visualization of Pit-1 transcription factor interactions in the living cell nucleus by fluorescence resonance energy transfer microscopy. Mol. Endocrinol., 12: 1410-1419.

De Grooth B.G. and Putman, C.J.A., 1992 - Highresolution imaging of chromosome-related structures by atomic force microscopy. Microscopy, 168: 239247.

De Rosier D.J. and KLug. A., 1968 - Reconstruction of three-dimensional structures from electron micrographs. Nature, 217: 130-132.

Deans S.R., 1983 - The Radon transform and some of its applications. Wiley, New York.

dos Remedios C.G., Miki M. and Barden J.A., 1987 - Fluorescence resonance energy transfer measurements of distances in actin and myosin: A critical evaluation. J. Muscle Res. Cell. Motil., 8: 97-117.

Elangovan M., Day R.N. and Periasamy A., $2002-$ Nanosecond fluorescence resonance energy transferfluorescence lifetime imaging microscopy to localize the protein interactions in a single living cell. J. Microsc., 205: 3-14.

Elangovan M., Wallrabe H., Chen Y., Day R.N., Barroso M. and Periasamy A., 2003 - Characterization of one-and two-photon excitation fluorescence resonance energy transfer microscopy. Methods, 29: 58-73.

Engel A., 1991 - Biological applications of scanning sensor microscopes. Ann. Rev. Biophys. Biophys. Chem., 20: 79-108.

ENGELHARDT P., 2000 - Electron tomography of chromosome structure. In: Encyclopaedia of Analytical Chemistry. Meyers RA, editor, pp. 4948-4984.

Earnshaw W.C. and Heck M.M., 1985 - Localization of topoisomerase II in mitotic chromosomes. J. Cell. Biol., 100: 1716-1725. 
Earnshaw W.C. and Laemmli U.K., 1983 - Architecture of metaphase chromosomes and chromosome scaffolds. J. Cell. Biol., 96: 84-93.

Earnshaw W.C. and Laemmli U.K., 1984 - Silver staining the chromosome scaffold. Chromosoma, 89: 186-192.

EHrenhofer-Murray AE., 2004 - Chromatin dynamics at DNA replication, transcription and repair. Eur. J. Biochem., 271: 2335-2349.

Förster T. 1965 - Delocalized excitation and excitation transfer. In: Modern Quantum Chemistry. Vol. 3, Sinanoglu O, editor. Academic Press Inc., New York, pp. 93-137.

Fotiadis D., Scheuring S., Muller S.A., Engel A. and Muller D.J., 2002 - Imaging and manipulation of biological structures with the AFM. Micron, 33: 385-397.

Fracke U., 1994 - Digitized and differentially shaded buman chromosome ideograms for genomic applications. Cytogenet. Cell. Genet., 65: 206-219.

FRANK J., 1992 - Electron tomography: three-dimensional imaging with the transmission electron microscopy. Plenum Press, New Work.

Giocondi M.C., Milhiet P.E., Lesniewska E. and Le Grimellec C., 2003 - Atomic force microscopy: from cellular imaging to molecular manipulation. Med Sci (Paris), 19: 92-99.

Harauz G., Borland L., Bahr G.F., Zeitler E. and vaN HeEl M., 1987 - Three-dimensional reconstruction of a buman metaphase chromosome from electron tomographas. Chromosoma, 95: 366-375.

Harnden D.G. and Klinger H.P., 1985 - An international system for buman cytogenetic nomenclature. Karger, Basel.

HART R., 1968 - Electron microscopy of unstained biological material: the polytropic montage. Science, 159: 1464-1467.

Heim R., Tsien R.Y., 1996 - Engineering green fluorescent protein for improved brightness, longer wavelengths and fluorescence resonance energy transfer. Curr. Biol., 6: 178-182

Heliot L., Kaplan H., Lucas L., Klein C., Beorchia A., Doco-Fenzy M., Menager M., Thiry M., O’Donohue M.F. and Ploton D., 1997 Electron tomography of metaphase nucleolar organizer regions: evidence for a twisted-loop organization. Mol. Biol. Cell., 8: 2199-2216.

Herman B., 1998 - Fluorescence Microscopy. 2nd ed. Springer-Verlag New York Inc., New York, USA.

Hoppe W., Langer R., Knesch G. and Poppe C., 1968 - Protein-kristallstrukturanalyse mit elektronenstrablen. Naturwissenschaften, 55: 333-336.

Horowitz R.A., Agard D.A., Sedat J.W. and WoodCOCK C.L., 1994 - The three-dimensional architecture of chromatin in situ: electron tomography reveals fibers composed of a continuously variable zig-zag nucleosomal ribbon. J. Cell. Biol., 125: 1-10.

Horowitz R.A., Koster A.J., Walz J. and Woodcock C.L., 1997 - Automated electron microscope tomography of frozen-hydrated chromatin: the irregular three-dimensional zigzag architecture persists in compact, isolated fibers. J. Struct. Biol., 120: 353 362.

Iwabuchit S., Mori T., Ogawa K., Sato K., Sato M., Morita Y., Ushiki R. and Tamiya Y., 2002 Atomic force microscope-based dissection of buman metaphase chromosomes and high resolutional imaging by carbon nano-tip. Arch. Histol. Cytol., 65: 473479.

Jack E.M., Harrison C.J., Allen T.D. and Harris R., 1986 - A structural basis for R- and T-banding: a scanning electron microscopy study. Chromosoma, 94: 395-402.

Jenkins E.C., Wen G.Y., Jenkins E.C. Jr., Genovese M. and Brown W.T., 2002 - Human chromatid ultra-structure: new observations with scanning and transmission electron microscopy. Scanning, 24: 55-58.

Klein C., Cheutin T., O’Donohue M.F., Rothblum L., Kaplan H., Beorchia A., Lucas L., Heliot L. and Ploton D., 1998 - The three-dimensional study of chromosomes and upstream binding factorimmunolabeled nucleolar organizer regions demonstrates their non-random spatial arrangement during mitosis. Mol. Biol. Cell., 9: 3147-3159.

Lakowicz J.R., 1999 - Principles of Fluorescence Spectroscopy. 2nd ed. Plenum Publishing Corp., New York. 692 pp.

Lichtman J.W., 1994 - Confocal microscopy. Sci. Am., 271: 40-45.

Mandrioli M. and Manicardi G.C., 2003 - Analysis of insect bolocentric chromosomes by atomic force microscopy. Hereditas, 138: 129-132.

McMaster T.J., Hickish T., Min T., Cunningham D. and Miles M.J. 1994 - Application of scanning force microscope to chromosome analysis. Cancer Genet. Cytogenetics, 76, pp. 93-95.

McMaster T.J., Winfield M.O., Karp A. and Miles M.J. 1996a - Analysis of cereal chromosomes by atomic force microscopy. Genome, 39: 439-444.

McNally J.G., Karpova T. and Cooper J. 1999 Three-Dimensional imaging by deconvolution microscopy. Methods, 19: 373-385.

Mochida K., Tsujimoto H.. and Sasakuma T. 2004 - Confocal analysis of chromosome behavior in wheat $x$ maize zygotes. Genome, 47: 199-205.

Musio A., Mariani T., Frediani C., Sbrana I. and Ascoli C. 1994 - Longitudinal patterns similar to G-banding in mammalian metaphase chromosomes. Chromosoma, 103: 225-229.

OCKLEFORD C. 1995 - The confocal laser scanning microscope (CLSM). J. Pathol., 176: 1-32.

Porter I.M., Khoudoli G.A. and Swedlow J.R. 2004 - Chromosome condensation: DNA compaction in real time. Curr. Biol., 14: 554-556.

RADON J. 1917 - Uber die Bestimmung von Funktionen durch ibre Intergralwerte langs gewisser Mannigfaltigkeiten. Ber Verh K Sachs Ges Wiss. Leipzig, Math-Phys Kl., 69: 262-277.

Rasch P., Widemann U., Winberg J. and Heckl W.M. 1993 - Analysis of banded buman chromosomes and in situ bybridisation patterns by scanning 
force microscopy. Proc. Natl. Acad. USA, 90: 25092511.

Rowland R.E. and Nickless E.M. 2000 - Confocal microscopy opens the door to $3 D$ analysis of cells. Bioscience, 26: 3-7.

Sanchez-Sweatman O.H., de Harven E.P. and Dubé I.D. 1993 - Human chromosomes evaluation of processing techniques for scanning electron microscopy. Scan. Microsc., 7: 97-106.

Santos A.P., Abranches R., Stoger E., Beven A., Viegas W. and Shaw P.J. 2002 - The architecture of interphase chromosomes and gene positioning are altered by changes in DNA methylation and histone acetylation. J. Cell Sci., 115: 4597-4605.

Schaper A., Rossle M., Formanek H., Jovin T.M. and Wanner G. 2000 - Complementary visualization of mitotic barley chromatin by field-emission scanning electron microscopy and scanning force microscopy. J. Struct. Biol., 129: 17-29.

Sekar R.B. and Periasamy A. 2003 - Fluorescence resonance energy transfer (FRET) microscopy imaging of live cell protein localizations. J. Cell Biol., 160: 629-633.

Shaw P.J., Abranches R., Paula Santos A., Beven A.F., Stoger E., Wegel E. and Gonzalez-MeLENDI P. 2003 - The architecture of interphase chromosomes and nucleolar transcription sites in plants. J. Struct. Biol., 140: 31-38.

Squarzoni S., Cinti C., Santi S., Valmori A. and MARALDi N.M. 1994 - Preparation of chromosome spreads for electron (TEM, SEM, STEM), light and confocal microscopy. Chromosoma, 103: 381-392.

Stark R.W., Rubio-Serra F.J., Thalhammer S. and HECKL W.M. 2003 - Combined nano-manipulation by atomic force microscopy and laser beam ablation for chromosomal dissection. Eur. Biophys. J., 32: 33 39.

STEVENS J.K. 1994 - Introduction to confocal three-dimensional volume investigaton. In: Three-dimensional confocal microscopy. STEvens JK, MiLls LR, Trogadis JE, editors. Academic Press, San Diego, USA.

Sugiyama S., Yoshino T., Kanahara H., Kobori T. and OHtani T. 2003 - Atomic force microscopic imaging of $30 \mathrm{~nm}$ chromatin fiber partially relaxed plant chromosomes. Scanning, 25: 132-136.

Sugiyama S., Yoshino T., Kanahara H., Shichiri M., Fukushi D. and Ohtani T. 2004 - Effects of acetic acid treatment on plant chromosome structures analyzed by atomic force microscopy. Anal. Biochem., 324: 39-44.

Sumner A.T. 1991 - Scanning electron microscopy of mammalian chromosomes from prophase to telophase. Chromosoma, 100: 410-418.

Sumner A.T. 1996 - Problems in preparation of chromosomes for scanning electron microscopy to reveal morphology and to permit immunocytochemistry of sensitive antigens. Scanning Microsc. Suppl., 10: 165-174.
Sumner A.T. 1998 - The structure of the centromeric region of $\mathrm{CHO}$ chromosomes. Cell. Biol. Int., 22: $127-130$

Sumner A.T. 2004 - Chromosomes: organization and function. Blackwell Publishing, Oxford, UK.

Sumner A.T, and Ross A. 1989 - Factors affecting preparation of chromosomes for scanning electron microscopy using osmium impregnation. Scanning Microsc., 3: 87-99.

Sumner A.T., Ross A.R. and Graham E. 1994 Preparation of chromosomes for scanning electron microscopy. Methods Mol. Biol., 29: 41-50.

Swedlow J.R. and Hirano T. 2003 - The making of the mitotic chromosome: modern insights into classical questions. Mol. Cell., 11: 557-569.

Tamayo J. 2003 a - Structure of buman chromosomes studied by atomic force microscopy. J. Struct. Biol., 141: 198-207.

TAmayo J. 2003b - Structure of buman chromosomes studied by atomic force microscopy. Part II. Relationship between structure and cytogenetic bands. J. Struct. Biol., 141: 189-197.

Tamayo J. and Miles M. 2000 - Human chromosome structure studied by scanning force microscopy after an enzymatic digestion of the covering cell material. Ultramicrosc., 82: 245-251.

Thalhammer S., Stark R.W., Muller S., Wienberg J. and HEckL W.M. 1997 - The atomic force microscope as a new microdissection tool for the generation of genetic probes. J. Struct. Biol., 119: 232-237.

Troster H., Spring H., Meissner B., Schultz P., Oudet P. and Trendelenburg M.F. 1985 - Structural organization of an active, chromosomal nucleolar organizer region (NOR) identified by light microscopy and subsequent TEM and STEM electron microscopy. Chromosoma, 91: 151-163.

Ushiki T., Hoshi O., Iwaki K., Kimura E. and ShigENO M. 2002 - The structure of human metaphase chromosome: its histological perspective and new borizon by atomic force microscopy. Arch. Histol. Cytol., 65: 377-390.

Wanner G. and Formanek H. 2000 - A new chromosome model. J. Struct. Biol., 132: 147-161.

Wanner G., Formanex H., Martin R. and Herrman R.G. 1991 - High resolution scanning electron microscopy of plant chromosomes. Chromosoma, 100: 103-109.

Weierich C., Brero A., Stein S., von Hase J., Cremer C., Cremer T. and Solovei I. 2003 - Threedimensional arrangements of centromeres and telomeres in nuclei of buman and murine lymphocytes. Chromosome Res., 11: 485-502.

WIESENDANGER R. 1994 - Scanning probe microscopy and spectroscopy: methods and applications. Cambridge University Press, UK.

Wilson P.J., Forer A. and Wise D. 2003 - Microtubule distribution during meiosis I in flea-beetle [Alagoasa (Oedionychus)] spermatocytes: evidence for direct connections between unpaired sex chromosomes. J. Cell Sci., 116: 1235-1247 
Winfield M., McMaster T.J., Karp A. and Miles M.J. 1995 - Atomic force microscopy of plant chromosomes. Chromosome Res., 3: 128-131.

Wolf K.W. and Sumner A.T. 1996 - Scanning electron microscopy of heterochromatin in chromosome spreads of male germ cells in Schistocerca gregaria (Acrididae, Orthoptera) after trypsinization. Biotech. Histochem., 71: 237-244.

Woodcock C.L. 1992 - The organization of chromosome and chromatin. In: Electron tomography. PLENUn J.F., editor. Academic Press, New York, pp 313-357.

Woodcock C.L. and Dimitrov S. 2001 - Higher-order structure of chromatin and chromosomes. Curr. Opin. Genet. Dev., 11: 130-135.
Wright S.J., Centonze V.E., Stricker S.A., Devries P.J., Paddock S.W. and Schatten G. 1993 - Introduction to confocal microscopy and three-dimensional reconstruction. Meth. Cell. Biol., 38: 38-56.

Xu X. and Wu M. 1983 - Electron microscopy of $G$-banded buman mitotic chromosomes.

Chromosoma, 88: 237-240.

Yang J., Tamm L.K., Somlyo A.P. and Shao Z. 1993 - Promises and problems of biological atomic force microscopy. J. Microsc., 171: 183-188.

Received 07.12.2005; accepted 11.02.2006 